\title{
FAR-INFRARED EXCITED HYDROXYL LINES FROM ORION KL OUTFLOWS ${ }^{1}$
}

\author{
Javier R. Goicoechea, ${ }^{2}$ José Cernicharo, ${ }^{3}$ Mercedes R. Lerate, ${ }^{4,5}$ Fabien Daniel, ${ }^{3}$ Michael J. Barlow, ${ }^{5}$ \\ Bruce M. Swinyard, ${ }^{4}$ Tanya L. Lim, ${ }^{4}$ Serena Viti, ${ }^{5}$ and Jeremy Yates ${ }^{5}$ \\ Received 2006 January 6; accepted 2006 March 2; published 2006 March 22
}

\begin{abstract}
As part of the first far-IR line survey toward Orion KL, we present the detection of seven new rotationally excited $\mathrm{OH} \Lambda$-doublets (at $\sim 48, \sim 65, \sim 71, \sim 79, \sim 98$, and $\sim 115 \mu \mathrm{m}$ ). Observations were performed with the Long Wavelength Spectrometer Fabry-Pérot on board the Infrared Space Observatory. In total, more than 20 resolved $\mathrm{OH}$ rotational lines, with upper energy levels up to $\sim 620 \mathrm{~K}$, have been detected at angular and velocity resolutions of $\sim 80^{\prime \prime}$ and $\sim 33 \mathrm{~km} \mathrm{~s}^{-1}$, respectively. The $\mathrm{OH}$ line profiles show a complex behavior evolving from pure absorption, P Cygni type, to pure emission. We also present a large-scale, $6^{\prime}$ declination raster in the $\mathrm{OH}^{2} \Pi_{3 / 2}$ $J=5 / 2^{+}-3 / 2^{-}$and ${ }^{2} \Pi_{3 / 2} J=7 / 2^{-}-5 / 2^{+}$lines (at 119.441 and $84.597 \mu \mathrm{m}$ ) revealing a decrease of excitation outside the core of the cloud. From the observed profiles, mean intrinsic line widths, and velocity offsets between emission and absorption line peaks, we conclude that most of the excited $\mathrm{OH}$ arises from Orion outflow(s), that is, the "plateau" spectral component. We determine an averaged $\mathrm{OH}$ abundance relative to $\mathrm{H}_{2}$ of $\chi(\mathrm{OH})=(0.5-$ 1.0) $\times 10^{-6}$, a kinetic temperature of $\gtrsim 100 \mathrm{~K}$, and a density of $n\left(\mathrm{H}_{2}\right) \simeq 5 \times 10^{5} \mathrm{~cm}^{-3}$. Even with these conditions, the $\mathrm{OH}$ excitation is heavily coupled with the strong dust continuum emission from the inner "hot core" regions and from the expanding flow itself.
\end{abstract}

Subject headings: infrared: ISM — ISM: individual (Orion KL) — ISM: jets and outflows — ISM: molecules — radiative transfer

\section{INTRODUCTION}

The Kleinmann-Low $(\mathrm{KL})$ infrared nebula $\left(L \sim 10^{5} L_{\odot}\right)$ in the Orion molecular cloud is the nearest $(\sim 450 \mathrm{pc})$ and probably the most studied massive star-forming region (Genzel \& Stutzki 1989). Early studies soon determined that the large-scale distribution of gas and dust is heavily influenced by violent phenomena such as the interaction of compact and large-scale outflows with the quiescent gas, producing strong line and continuum emission. IRc2 was believed to be the main source of luminosity, heating, and dynamics in the region. However, great advances in near- and mid-IR subarcsecond resolution imaging and in (sub-) millimeter interferometric observations have dramatically changed our understanding of the region. First, the $8-12 \mu \mathrm{m}$ emission peak of IRc2 is not coincident with the Orion $\mathrm{SiO}$ maser origin (related to the origin of the outflow or outflows), and second, its intrinsic IR luminosity $\left(L \sim 1000 L_{\odot}\right)$ is only a fraction of the complex luminosity (Gezari et al. 1998). In addition, 3.6-22 $\mu \mathrm{m}$ images show that IRc2 is in fact resolved into four components that may even not be self-luminous. Therefore, the relevance of IRc2 as the powerful engine of Orion KL is no longer supported, and its nature is now even less clear (Dougados et al. 1993; Shuping et al. 2004; Greenhill et al. 2004). A new step forward was taken by Menten \& Reid (1995) with the detection of the very embedded radio continuum source "I" (located 0".5 south of IRc2), which coincides with the centroid of the $\mathrm{SiO}$ maser

\footnotetext{
${ }^{1}$ Based on observations with $I S O$, an ESA project with instruments funded by ESA member states (especially the PI countries: France, Germany, the Netherlands, and the UK) and with the participation of ISAS and NASA.

${ }^{2}$ Laboratoire d'Etude du Rayonnement et de la Matière en Astrophysique, UMR 8112, CNRS, Ecole Normale Supérieure and Observatoire de Paris, 24 rue Lhomond, F-75231 Paris Cedex 5, France; javier@1ra.ens.fr.

${ }^{3}$ Departamento de Astrofísica Molecular e Infrarroja, Instituto de Estructura de la Materia, CSIC, Calle Serrano 121, E-28006 Madrid, Spain.

${ }^{4}$ Rutherford Appleton Laboratory, Chilton, Didcot OX11 0QX, UK.

${ }^{5}$ Department of Physics and Astronomy, University College London, Gower Street, London WC1E 6BT, UK.
}

distribution. Menten \& Reid also detected the radio continuum emission of IR source "n" and suggested that it could also contribute to the origin of some of the phenomena observed at larger scales. Thus, in addition to BN, the core of Orion KL contains at least two more compact $\mathrm{H}$ II regions (I and $\mathrm{n}$ ) that seem to be running away from a common point, suggesting that $\mathrm{BN}$, I, and $\mathrm{n}$ were originally part of a common massive stellar system that disintegrated $\sim 500$ years ago (Gómez et al. 2005). A causal relation between the dynamical decay of this stellar system and the observed large-scale molecular outflow(s), $\mathrm{H}_{2}$ fingers, and bow shocks (Stolovy et al. 1998) has been proposed (Bally \& Zinnecker 2005).

The different physical conditions and velocity fields along the line of sight result in a segregation of the gas and dust chemistry that complicates the interpretation of observations toward Orion, especially for low angular resolution molecular surveys. For this reason, it is common to distinguish between different spectral components: the "ridge" (extended quiescent molecular gas), the "hot core" (a collection of very dense and hot gas clumps in which sources I and $\mathrm{n}$ are embedded), and the "plateau" (a mixture of outflow[s], shocks, and interactions with the ambient cloud). Submillimeter aperture synthesis line surveys have finally provided the spatial location and extent of many molecular species (Blake et al. 1996; Wright et al. 1996; Liu et al. 2002; Beuther et al. 2005). As a result, the formation of complex oxygen-bearing species in the interaction region between the outflow(s) and the quiescent gas is now generally understood in the context of an oxygen-rich molecular outflow (Blake et al. 1987; Wright et al. 1996; Liu et al. 2002). However, the thermal lines from basic oxygen reservoirs $\left(\mathrm{O}, \mathrm{H}_{2} \mathrm{O}\right.$, and $\left.\mathrm{OH}\right)$ lie in the far-IR, a spectral region blocked by Earth's atmosphere. Besides, the wide range of excitation conditions provided by oxygen hydrides supply unique information about the energetics and dynamics of the region. In particular, far-IR OH line emission (first detected by Storey et al. 1981) is predicted to be a powerful diagnostic of the plateau gas (Draine \& Roberge 1982). Observations with the Kuiper 

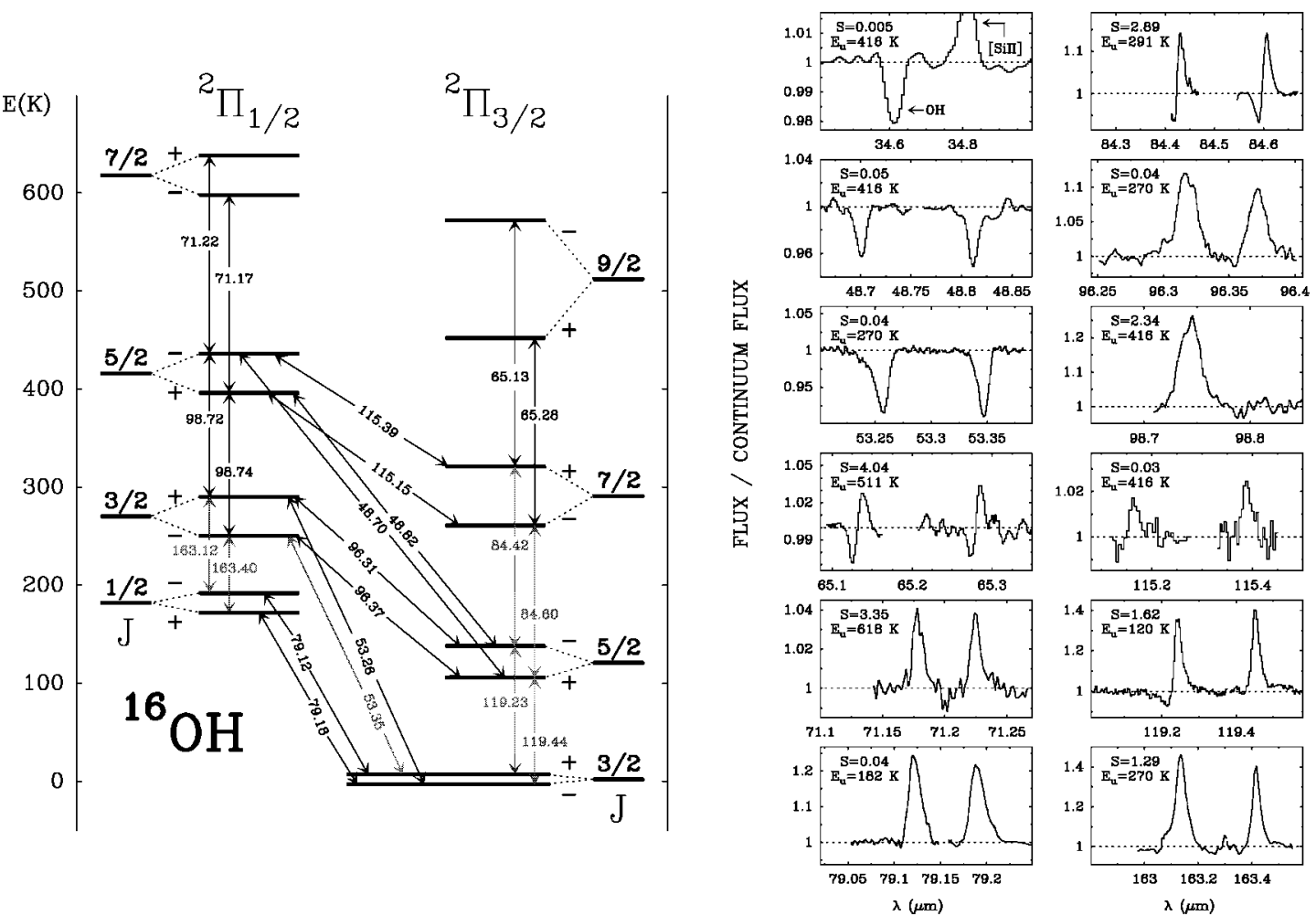

FIG. 1.-Left: Rotational energy diagram of ${ }^{16} \mathrm{OH}$ showing the lines detected by $I S O$ (in microns). Newly detected transitions are shown in black, while previous KAO detections are shown in gray. The ${ }^{2} \Pi_{3 / 2}$ and ${ }^{2} \Pi_{1 / 2}$ rotational ladders produced by the spin-orbit interaction are shown. The $\Lambda$-doubling splitting of each rotational level has been enhanced for clarity. Hyperfine structure is not shown. Right: ISO-LWS-FP observations of ${ }^{16} \mathrm{OH}$ toward Orion. The vertical axes correspond to the continuum-normalized flux and the horizontal axes to the wavelength in microns. In each panel, the upper level energy (in kelvins) and the intrinsic line strength of the transition are also shown. The upper left panel corresponds to the unresolved $\mathrm{OH}^{2} \Pi_{3 / 2}-{ }^{2} \Pi_{1 / 2} J=3 / 2-5 / 2$ lines at $\sim 34 \mu \mathrm{m}$ detected by the $I S O$ Short Wavelength Spectrometer (Wright et al. 2000).

Airborne Observatory (KAO) allowed the detection of several low-excitation $\mathrm{OH}$ rotational lines, as illustrated in Figure 1 (Watson et al. 1985; Viscuso et al. 1985; Betz \& Boreiko 1989; Melnick et al. 1987, 1990). The specific contributions of the hot core, plateau, and ridge components and the physical conditions within the $\mathrm{OH}$-emitting gas remain open to question.

\section{OBSERVATIONS AND DATA REDUCTION}

Most of the $\mathrm{OH}$ pure rotational lines appear in the wavelength range of the Infrared Space Observatory (ISO) Long Wavelength Spectrometer (LWS; Clegg et al. 1996). The size of the LWS circular aperture is $\sim 80^{\prime \prime}$. In its Fabry-Pérot (FP) mode, the instrumental response is close to a Lorentzian with a spectral resolution of $\sim 33 \mathrm{~km} \mathrm{~s}^{-1}$. In this Letter, we present observations that are part of the first full far-IR line survey of Orion $\mathrm{KL}$ using the unprecedented $I S O$-LWS-FP wavelength coverage and velocity resolution (Lerate et al. 2006). OH spectra were obtained in the Astronomical Observation Template (AOT) L04 and L03 modes. Processing of the $\mathrm{OH}$ spectra from AOT L03 was carried out using the Off-Line Processing (OLP) pipeline and the LWS Interactive Analysis (LIA) package, version 10. AOT L04 spectra were analyzed using the ISO Spectral Analysis Package (ISAP). ${ }^{6}$ Typical routines include dark current optimization, deglitching and removal of the LWS grating profile, oversampling and averaging individual scans, baseline removal, and line flux measurements. A full description of the

\footnotetext{
${ }^{6}$ ISAP is a joint development by the LWS and SWS Instruments Teams and Data Centers. Constributing institutes are CESR, IAS, IPAC, MPE, RAL, and SRON
}

complex data calibration and reduction process and associated Target Dedicated Time numbers (TDTs) of the survey are given in Lerate et al. (2006). The resulting OH lines are shown in Figure 1. Finally, we present a $\pm 180^{\prime \prime}$ declination raster (see Fig. 2) in the $\mathrm{OH}^{2} \Pi_{3 / 2} J=5 / 2^{+}-3 / 2^{-}$and ${ }^{2} \Pi_{3 / 2} J=7 / 2^{-}-$ $5 / 2^{+}$lines at 119.441 and $84.597 \mu \mathrm{m}$ (L04, TDT 70101216).

\section{RESULTS AND DISCUSSION}

The far-IR OH lines exhibit complicated behavior, evolving from pure absorption to pure emission profiles. The detection of P Cygni type profiles in ${ }^{2} \Pi_{3 / 2} \mathrm{OH}$ excited rotational lines at $\sim 65$ and $\sim 84 \mu \mathrm{m}$ (Fig. 1) and in the ${ }^{18} \mathrm{OH}$ ground-state line (Fig. 2; also detected by KAO [Melnick et al. 1990]) confirms that most of the $\mathrm{OH}$ emission arises from an extended molecular outflow. Since its optical depth is small, the ${ }^{18} \mathrm{OH}$ emission helps to trace the dominant outflow origin of the $\mathrm{OH}$ emission. Compared with stellar wind profiles, the molecular far-IR P Cygni profiles are favored by the presence of far-IR (dust) continuum emission throughout the expanding flow (and not only from a central continuum source). Hence, the detailed balance between emission and absorption components critically depends on the dust and $\mathrm{OH}$ emissivities, opacities, and beam filling factors at each wavelength. Simple nonlocal nonLTE radiative transfer models for dust and $\mathrm{OH}$ easily reproduce these characteristics (see below). On the other hand, all observed $\mathrm{OH}$ lines within the ${ }^{2} \Pi_{1 / 2}$ ladder $(\sim 71, \sim 98$, and $\sim 163 \mu \mathrm{m})$ appear in emission with a peak velocity of $v \gtrsim$ $+15 \mathrm{~km} \mathrm{~s}^{-1}$, showing less indication of self-absorption (the cloud rest velocity is $\sim 9 \mathrm{~km} \mathrm{~s}^{-1}$; Scoville et al. 1983). Taking into account the high energy of the associated levels, effective 

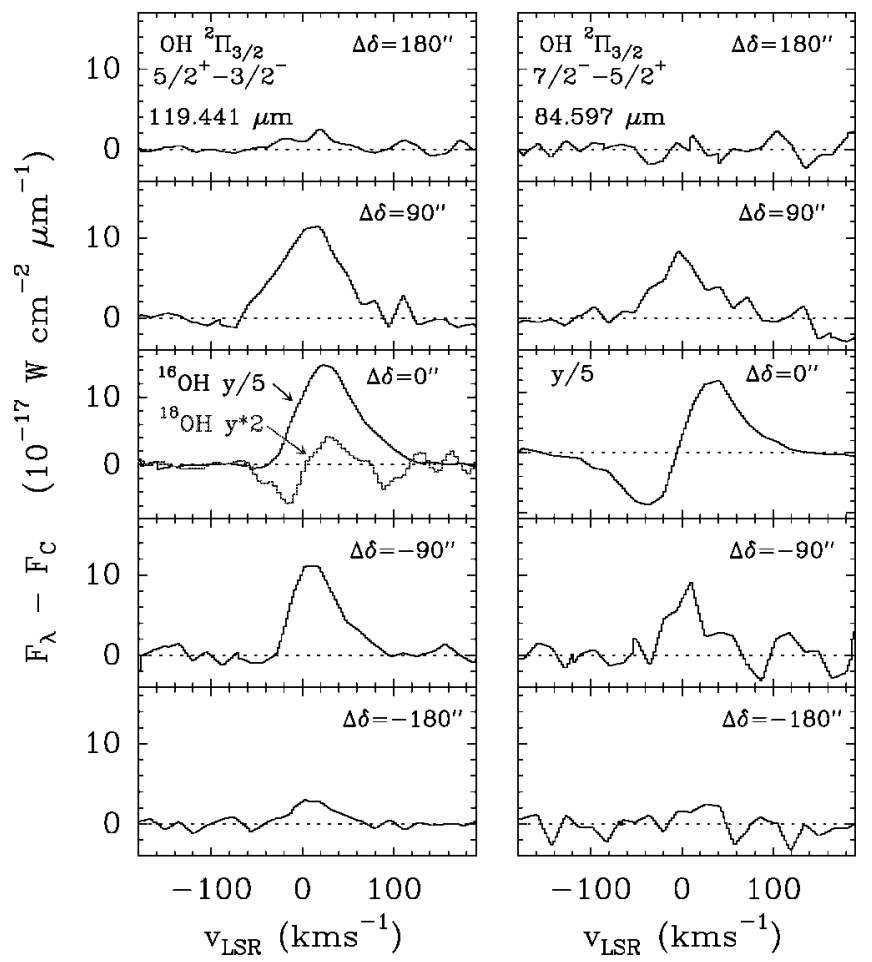

FIG. 2.-Continuum-subtracted ISO-LWS-FP spectra taken at different declination offsets from Orion IRc2. Offsets in arcseconds are indicated. The left $\left(0^{\prime \prime}, 0^{\prime \prime}\right)$ panel shows the different ground-state line profiles produced by ${ }^{16} \mathrm{OH}$ and ${ }^{18} \mathrm{OH}$ (also detected by $\mathrm{KAO}$ ).

radiative pumping by the far-IR dust continuum emission must be playing a role in the $\mathrm{OH}$ excitation. This is clearly favored by the detection of several ${ }^{2} \Pi_{3 / 2}-\Pi_{1 / 2}$ cross-ladder transitions in almost pure absorption $(\sim 34, \sim 48$, and $\sim 53 \mu \mathrm{m})$. Hence, temperatures and densities in the outflow can be significantly lower than those required to thermalize the $\mathrm{OH}$ emission lines. Other newly detected $\mathrm{OH}$ excited cross-ladder transitions ( 96 and $\sim 115 \mu \mathrm{m}$ ) are observed in pure emission, and they contribute to the radiative de-excitation of associated ${ }^{2} \Pi_{1 / 2}$ upper levels. Note that the absorption peak of pure absorption $\mathrm{OH}$ lines occurs at negative velocities, $v \lessgtr-15 \mathrm{~km} \mathrm{~s}^{-1}$. Similar conclusions apply for water lines (Cernicharo et al. 1999b). In particular, pure absorption mid-IR $\mathrm{H}_{2} \mathrm{O}$ lines peak at $-8 \pm$ $3 \mathrm{~km} \mathrm{~s}^{-1}$ (Wright et al. 2000). Taking into account the velocity resolution and wavelength calibration of the LWS/FP instrument, we find $25 \pm 5 \mathrm{~km} \mathrm{~s}^{-1}$ as the most likely expansion velocity of the far-IR OH flow.

The inferred expansion velocity seems more consistent with the $18 \pm 2 \mathrm{~km} \mathrm{~s}^{-1}$ low-velocity outflow, originally revealed by water maser motions (Genzel et al. 1981). In addition, it is known that $\mathrm{OH} 1.6 \mathrm{GHz}$ masers toward the Orion KL region are detected over an area of $30^{\prime \prime}$ in diameter. The best kinematic model fitting the $\mathrm{OH}$ maser emission is found for a uniform expansion velocity of $21.0 \pm 3.5 \mathrm{~km} \mathrm{~s}^{-1}$ away from a source (at or near IRc2) with a radial velocity of $9.0 \pm 0.5 \mathrm{~km} \mathrm{~s}^{-1}$ (Cohen et al. 2006). Contribution from the extended highvelocity outflow (Martín-Pintado et al. 1990) could also be present (see predictions by Melnick et al. 1990). Only the ${ }^{16} \mathrm{OH}$ ground-state lines at $\sim 119 \mu \mathrm{m}$ show high-velocity broad red wing emission above $\sim 100 \mathrm{~km} \mathrm{~s}^{-1}$. This high-velocity emission is not observed in any other $\mathrm{OH}$ line at the ISO observations' sensitivity. The large-scale $\mathrm{OH}$ declination raster clearly shows that $\mathrm{OH}$ lines are weaker (at least by a factor of 5) outside the

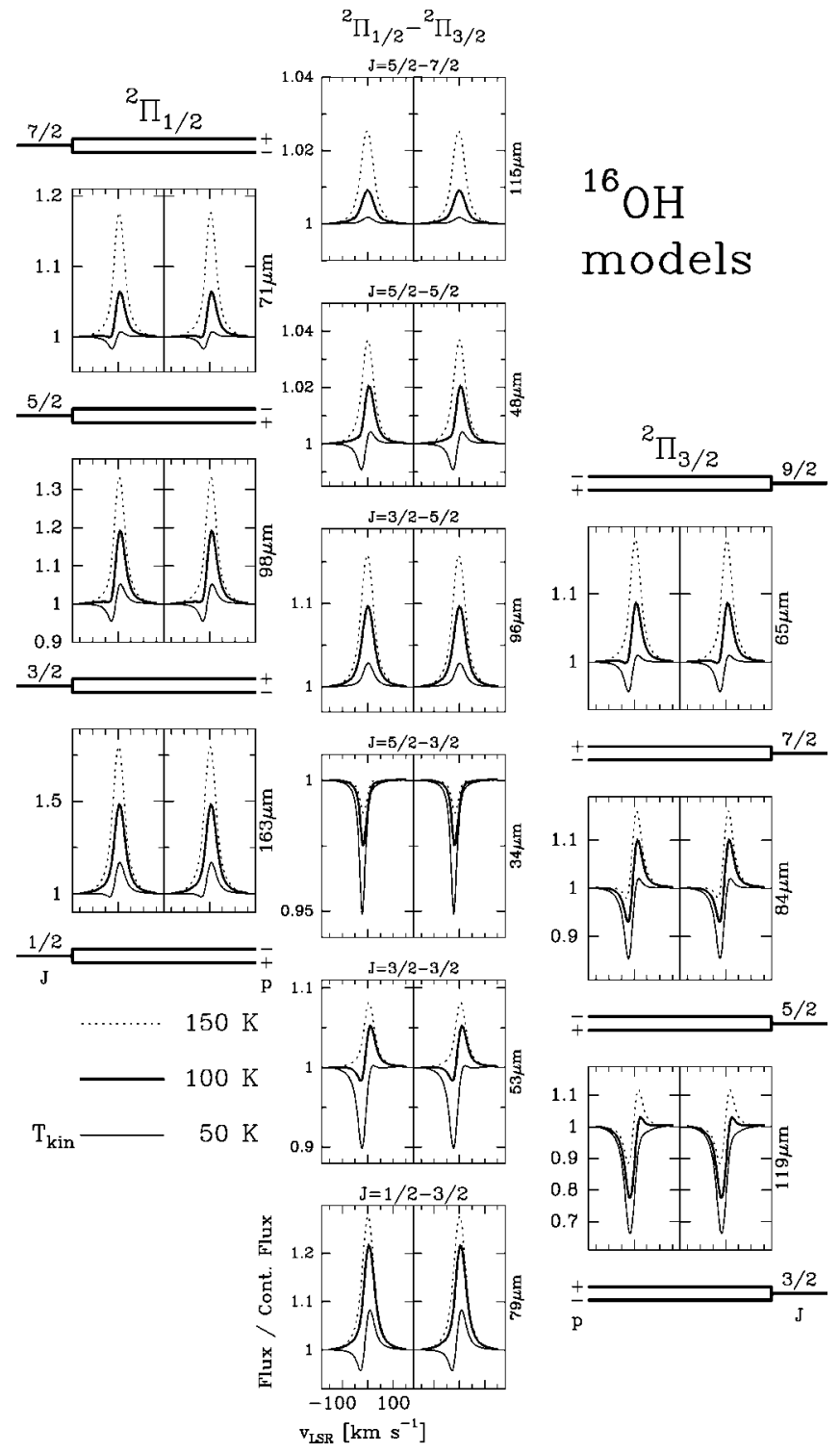

FIG. 3.-Selected radiative transfer models for an expanding OH shell. Common parameters are $\chi(\mathrm{OH})=5 \times 10^{-7}$ and $n\left(\mathrm{H}_{2}\right)=5 \times 10^{5} \mathrm{~cm}^{-3}$. Three different temperatures are considered: $T_{k}=50,100$, and $150 \mathrm{~K}$. Each panel corresponds to a rotational $\Lambda$-doublet. The wavelength of each transition is shown. Line profiles have been convolved with a Gaussian beam of $80^{\prime \prime}$ and with a Lorentzian of $33 \mathrm{~km} \mathrm{~s}^{-1}$.

central position (Fig. 2). This is likely due to lower excitation (continuum emission and densities decrease) in the extended cloud, but not necessarily to a steep decrease of $\mathrm{OH}$ abundance in the ridge. Unfortunately, it is impossible to infer the exact spatial distribution of the newly detected $\mathrm{OH}$ lines from the large ISO beam. KAO heterodyne observations of the $\sim 119.2 \mu \mathrm{m}$ line (with a beam of $\sim 33^{\prime \prime}$ ) showed that the $\mathrm{OH}$ emission may be spatially compact. Betz \& Boreiko (1989) deduced that the $\mathrm{OH}$ emission comes from a source less than $25^{\prime \prime}$ in FWHM diameter and, therefore, the same inner regions of the large-scale outflow revealed by $\mathrm{H}_{2} \mathrm{O}$ masers at $\sim 325 \mathrm{GHz}$ (Cernicharo et al. 1999a), and HDO emission at $\sim 893$ and $\sim 850 \mathrm{GHz}$ (Pardo et al. 2001). Besides, OH may also arise from the regions where the outflow plunge into the quiescent cloud, producing dissociative shocks. According to recent [C I] observations (with 10" angular resolution), molecular dissociation occurs in a shell of $\sim 40^{\prime \prime}$ diameter (Pardo et 
al. 2005) and, therefore, within the LWS beam. Nevertheless, the OH emission measured by Betz \& Boreiko (1989) toward the bright $\mathrm{H}_{2}$ shocked regions "peak 1" and "peak 2" is significantly weaker than toward the outflow itself. More quantitative conclusions about the spatial distribution of the $\mathrm{OH}$ flow require much higher angular resolution observations.

In order to estimate the $\mathrm{OH}$ abundance and the physical conditions leading to the observed line profiles, we have modeled the first $20 \mathrm{OH}$ rotational levels with the nonlocal code used in our OH study of Sgr B2 (Goicoechea \& Cernicharo 2002). We have modeled a spherical shell with a $25^{\prime \prime}$ diameter expanding at $25 \mathrm{~km} \mathrm{~s}^{-1}$ that surrounds a uniform core, optically thick in the far-IR, with a $10^{\prime \prime}$ diameter and a color temperature of $150 \mathrm{~K}$. These values agree with those obtained from highdipole molecules toward the hot core. In all models, the dust emission reasonably fits the far-IR continuum levels measured by LWS. Obviously, this model simplifies the inhomogeneous and clumpy nature of the region, as ISO observations average all the substructure morphology and the different physical conditions found in the plateau. In our view, the far-IR OH excited emission may be spatially associated with the $\mathrm{SO}$ and $\mathrm{SO}_{2}$ shell of low-velocity expanding gas, observed at $\sim 1^{\prime \prime}$ resolution, where the outflow shocks dense clumps of ambient material (Wright et al. 1996). A grid of models has been generated by varying $T_{k}=T_{\text {dust }}$ from 50 to $250 \mathrm{~K}, n\left(\mathrm{H}_{2}\right)$ from $10^{5}$ to $10^{7} \mathrm{~cm}^{-3}$, and $\chi(\mathrm{OH})$ from $10^{-8}$ to $10^{-5}$ in the outflow. The newly detected $\mathrm{OH}$ excited lines add some important constraints to the plateau physical conditions. Basically, the fact that excitation temperatures in several excited $\mathrm{OH}$ cross-ladder transitions have to be below $T_{\text {dust }}$ constrains the maximum $T_{k}$ and $n\left(\mathrm{H}_{2}\right)$ allowed to produce absorption in these transitions. These absorptions can only be reproduced if $n\left(\mathrm{H}_{2}\right)<5 \times$ $10^{6} \mathrm{~cm}^{-3}$; otherwise, collisional excitation and reemission dominates. For this range of $n\left(\mathrm{H}_{2}\right)$, the bulk of gas has to be above $T_{k}=50 \mathrm{~K}$, or else absorption will dominate the lower energy cross-ladder and ${ }^{2} \Pi_{3 / 2}$ intraladder transitions. On the other hand, collisional excitation will dominate for $T_{k}>200 \mathrm{~K}$. Since conditions of high $T_{k}$ and $n\left(\mathrm{H}_{2}\right)$ result in a pure $\mathrm{OH}$ emission-line spectrum, a dominant contribution from the hot core is not expected. In addition, the large far-IR line-plus-continuum opacity in the plateau itself will hide most of the hot core $\mathrm{OH}$ emission. The lack of spectral resolution makes a more accurate analysis of possible absorptions impossible.

Nevertheless, the detected $\mathrm{OH}$ self-absorptions and P Cygni type profiles allow one to constrain the physical parameters that lead to the observed balance between collisions and radiative pumping. The best single-component fit to the $\mathrm{OH}$ observations is obtained around $T_{k} \sim 100 \mathrm{~K}$ and $n\left(\mathrm{H}_{2}\right) \sim 5 \times 10^{5} \mathrm{~cm}^{-3}$ (see models in Fig. 3). In comparison with far-IR $\mathrm{H}_{2} \mathrm{O}$ groundstate or CO high- $J$ lines, $\mathrm{OH}$ cross-ladder transitions have both small spontaneous emission rates and small line strengths. In most applications, this indicates that the associated far-IR lines are optically thin. The lack of opacity broadening also implies that their line profiles are more sensitive to gas velocity fields and turbulence. A good agreement with observations is found for $\chi(\mathrm{OH})=(0.5-1.0) \times 10^{-6}$. Therefore, ISO observations clearly show that $\mathrm{OH}$ is very abundant in the plateau. These results provide additional insights into the fact that complex oxygen-rich molecules such as methanol $\left(\mathrm{CH}_{3} \mathrm{OH}\right)$ (Wright et al. 1996) or formic acid (HCOOH) (Liu et al. 2002) are specifically enhanced (when resolved at much higher resolution) in the interaction surfaces between the outflow (OH-rich) material and the ambient cloud. Only the wide range of excitations provided by water lines and the input from chemical models, able to predict the chemistry evolution of the physically distinct regions in Orion, can extract more accurate information from far-IR observations.

We are grateful to the LWS instrument team and the ISO database team for the quality of the provided data. J. R. G. was supported by a Marie Curie Intra-European Individual Fellowship within the Sixth European Community Framework Programme, contract MEIF-CT-2005-515340. We acknowledge the Spanish DGES and PNIE grants ESP 2001-4516 and AYA 2003-2785.

\section{REFERENCES}

Bally, J., \& Zinnecker, H. 2005, AJ, 129, 2281

Betz, A. L., \& Boreiko, R. T. 1989, ApJ, 346, L101

Beuther, H., et al. 2005, ApJ, 632, 355

Blake, G. A., Sutton, E. C., Masson, C. R., \& Phillips, T. G. 1987, ApJ, 315, 621

Blake, G. A., Mundy, L. G., Carlstrom, J. E., Padin, S., Scott, S. L., Scoville, N. Z., \& Woody, D. P. 1996, ApJ, 472, L49

Cernicharo, J., Pardo, J. R., González-Alfonso, E., Serabyn, E., Phillips, T. G., Benford, D. J., \& Mehringer, D. 1999a, ApJ, 520, L131

Cernicharo, J., et al. 1999b, in The Universe as Seen by ISO, ed. P. Cox \& M. F. Kessler (ESA-SP 427) (Noordwijk: ESA), 565

Clegg, P. E., et al. 1996, A\&A, 315, L38

Cohen, R. J., Gasiprong, N., Meaburn, J., \& Graham, M. F. 2006, MNRAS, 367,541

Dougados, C., Léna, P., Ridgway, S. T., Christou, J. C., \& Probst, R. G. 1993, ApJ, 406, 112

Draine, B. T., \& Roberge, W. G. 1982, ApJ, 259, L91

Genzel, R., Reid, M. J., Moran, J. M., \& Downes, D. 1981, ApJ, 244, 884

Genzel, R., \& Stutzki, J. 1989, ARA\&A, 27, 41

Gezari, D. Y., Backman, D. E., \& Werner, M. W. 1998, ApJ, 509, 283

Goicoechea, J. R., \& Cernicharo, J. 2002, ApJ, 576, L77

Gómez, L., Rodríguez, L. F., Loinard, L., Lizano, S., Poveda, A., \& Allen, C. 2005, ApJ, 635, 1166
Greenhill, L. J., Gezari, D. Y., Danchi, W. C., Najita, J., Monnier, J. D., \& Tuthill, P. G. 2004, ApJ, 605, L57

Lerate, M. R., et al. 2006, MNRAS, submitted

Martín-Pintado, J., Rodríguez-Franco, A., \& Bachiller, R. 1990, ApJ, 357, L49

Melnick, G. J., Genzel, R., \& Lugten, J. B. 1987, ApJ, 321, 530

Melnick, G. J., Stacey, G. J., Genzel, R., Lugten, J. B., \& Poglitsch, A. 1990, ApJ, 348, 161

Menten, K. M., \& Reid, M. J. 1995, ApJ, 445, L157

Liu, S.-Y., Girart, J. M., Remijan, A., \& Snyder, L. E. 2002, ApJ, 576, 255

Pardo, J. R., Cernicharo, J., Herpin, F., Kawamura, J., Kooi, J., \& Phillips, T. G. 2001, ApJ, 562, 799

Pardo, J. R., Cernicharo, J., \& Phillips, T. G. 2005, ApJ, 634, L61

Scoville, N., Kleinmann, S. G., Hall, D. N. B., \& Ridgway, S. T. 1983, ApJ, 275, 201

Stolovy, S. R. et al. 1998, ApJ, 492, L151

Storey, J. W. V., Watson, D. M., \& Townes, C. H. 1981, ApJ, 244, L27

Shuping, R. Y., Morris, M., \& Bally, J. 2004, AJ, 128, 363

Viscuso, P. J., Stacey, G. J., Harwit, M., Haas, M. R., Erickson, E. F., \& Duffy, P. B. 1985, ApJ, 296, 149

Watson, D. M., Genzel, R., Townes, C. H., \& Storey, J. W. V. 1985, ApJ, 298, 316

Wright, C. M., van Dishoeck, E. F., Black, J. H., Feuchtgruber, H., Cernicharo, J., González-Alfonso, E., \& de Graauw, T. 2000, A\&A, 358, 689

Wright, M. C. H., Plambeck, R. L., \& Wilner, D. J. 1996, ApJ, 469, 216 